\title{
Numerical study of heat flow characteristics of turbulent Taylor-Couette flow in slit wall model
}

\author{
Dong Liu*, Mohammed Ibrahim Abdelrahman Mohammedsalih, Amponsah-Gyenin Nana \\ Kofi, Shi-cheng Ding and Si-liang Sun
}

School of Energy and Power Engineering, Jiangsu University, Zhenjiang, 212013, China

* Corresponding author: liudong@ujs.edu.cn

$\begin{array}{ll}\text { Submitted } & : 16 / 03 / 2020 \\ \text { Revised } & : 02 / 11 / 2020 \\ \text { Accepted } & : 12 / 11 / 2020\end{array}$

\begin{abstract}
Heat transfer enhancement is by far an important component in the design of numerous industrial applications of Taylor-Couette flow including electric motors and particularly rotating machinery. To optimize the performances of these machines, superior knowledge of the fluid flow is vital to better estimate the heat transfer distribution. This study will specifically consider the effect the slit number and width possess on the distribution of turbulent TaylorCouette flow and the resulting heat transfer correlation in the annulus of two concentric cylinders under varying conditions. A numerical simulation method is intended for the study using varying slit structure parameters of widths $(2.5 \leq w \leq 7.5) \mathrm{mm}$ and fitted with 6,9 , and 12 number of slits. The slit effect is then investigated under both isotherm and non-isotherm conditions considering the interactions between fluid flow regions in the mainstream area and the annulus. The small-scale vortex that appears in the annulus region improves the heat transfer ability between the fluid in the annulus and the main region as well as the heat transfer performance of the model with a gradual increase in Reynolds number.
\end{abstract}

Keywords: Heat transfer; Numerical method; Slit wall geometry; Taylor-couette flow; Turbulence flow.

\section{INTRODUCTION}

The forced convective heat transfer and turbulent problem in the annulus located between two concentric cylinders, with both or one of them rotating, has several engineering applications in industry, such as cooling of rotating machines, treatments of liquid metals, rotating tube heat transfer, chemical vapor deposition, and nuclear reactors (Kreith, 1968, Singer, 1984, Vivès 1988, and Selimefendigil et al., 2014). Besides these practical concerns, this configuration model has a significant classical problem in heat transfer and fluid flow. A lot of studies conducted by researches, observed in this flow model, have extensive information that accumulated in literature along past years, for which a good review articles about the state of the art of fluid flow and heat transfer between concentric rotating cylinders are now available (see Fénot et al., 2011, for more information about this topic). The conventional industrial coolants like water, air, oils, and others have less performance of heat transfer in most engineering applications, which returns to the low thermal conductivity of these types of fluids. Hence, we could find many experimental and numerical investigations research in the direction of the developing configuration geometries in the thermal systems to enhance heat transfer performance. 
The flow dynamics and heat transfer in annulus between two concentric rotating cylinders without axial flow are recognized with Taylor-Couette flow. In rotating machineries, a perfect knowledge about the hydrodynamics and heat transfer characteristics is required to give a good predict of the heat transfer distribution and thus to optimize the performances of the machine parts. During the operation of some mechanical and thermal systems, the temperature is arising to alarming rates. That makes a negative effect on the manufactured materials, which can lead to mechanical failure or thermoplasticization. These types of problems motivate several researchers to study how to get the enhanced heat transfer in cylindrical annulus gap flow between two concentric cylinders. For instance, Yong and Minkowycz (1989) experimentally investigated the heat transfer characteristics in annulus between two concentric cylinders, in the presence of grooves on the wall of inner one. They found that, from their results, the grooves on the wall of rotating inner cylinder can increase $\mathrm{Nu}_{j}$ (Nusselt number) than that over the smooth cylinder. Hayase et al. (1992) numerically analyzed the flow dynamics and heat transfer characteristics in Taylor-Couette model with non-smooth cylindrical gap, in slotted wall. They observed that the cavities which are embedded in the inner cylinder wall have an influence in enhancing heat transfer. Also, according to a study by Lopez et al. (2015), which considered the distribution of the flow field under different flow conditions, it was revealed that the axial length of the model, ring gap width $d$, Rayleigh, and Reynolds numbers have an important influence on the flow field rotation and thermal behavior.

In turbulent Taylor-Couette flow regime, many studies have been conducted by both experimental and numerical investigations (Gollub \& Swinney, 1975; Alziary \& Grillaud, 1978). Ostilla-Monico et al. (2014) carried out work, in which they used direct numerical simulations (DNS) to explore Taylor-Couette flow in a fully turbulent flow state. The study showed that the flow field from the turbulent Taylor-Couette flow to the fully turbulent flow is influenced by factors such as the model aspect ratio, speed, and radius. In the form of V-shape walls grooves on the inner and outer cylinders, Zhu et al. (2016) have investigated numerically turbulent Taylor-Couette flow. They postulated an important interpretation stating that the relationship between the momentum transport and the existence of the wall grooves. Several other authors have delved into Taylor number (Ta), by increasing or decreasing it, resulting in flow evolution and heat transport (Coles, 1965; Serre et al., 2008).

Moreover, experimental and numerical studies of slit wall configuration geometry influence on flow distribution and heat transfer within a Taylor-Couette system have surged due to the importance of increased heat transfer rate within a cylindrical annulus gap. The study by Jeng et al. (2008) aimed at determining the heat augmentation in the annulus gap with an inner rotating rib roughness cylinder. The results showed that the cylinder ribbed surface improved the heat transfer compared to the plain model. Liu et al. (2015) numerically probed into the effect of the introduction of the slit on the heat transfer in Taylor vortex flow. The results from their work revealed a $14.2 \%$ increase by calculating the heat flux generated by the 12-slit model inner cylinder, and this model has a better heat transfer ability in comparison to the smooth model. The turbulent Taylor-Couette flow in slit model was numerically studied by Liu et al. (2019), and the result showed that the velocity in the slit area is consistent with the change of wall shear force, but the heat flux is opposite. Experimentally, a study by Nouri-Borujerdi et al. (2017) investigated the effect of the presence of slits on heat transfer and pressure drop in a concentric cylinder. The result showed correlations for Nusselt number and the pressure drop in terms of the axial Reynolds number, Taylor number, the number of slits, and the slit aspect ratio influencing the heat transfer ability of the system.

Among the above researches, the complexity of cylinders walls structure and heat generation in the annulus region in most engineering problems cases makes the flow within the annulus contrary to that of the plain wall. The effect of slit wall has been noted in some literature but in the author's knowledge, a systematic study on its combined impact on the annulus flow field distribution and heat transfer performance has rarely been factored. This will be considered by analyzing the heat flow characteristics in a turbulent Taylor-Couette model under both isothermal and non-isothermal configurations. Also, the influence of the slits numbers and width of the respective models on convection field distribution and heat transfer augmentation is investigated to provide a reference for the design and 
optimization of related rotating machinery in an attempt to solve the engineering problem. Results from the study generally predict an increase in the heat transfer performance of the model when both slit number and the average Nusselt number increase.

\section{CALCULATION PARAMETERS AND MATHEMATICAL MODEL}

\section{Model Describtion}

The schematic diagram of slit model is shown in Fig. 1 . The inner cylinder has a radius of $\mathrm{ri}=33 \mathrm{~mm}$ and the outer cylinder has a radius of ro $=40 \mathrm{~mm}$. Hence, the gap between the two cylinders is $d=7 \mathrm{~mm}$. The length of the cylinder is $\mathrm{L}=336 \mathrm{~mm}$. The heat transfer areas of the different models are the same, which ensures that the heat transfer process of the models is not affected. The definition of the equivalent diameter of the two concentric cylinder model is

$$
D_{h}=\frac{2\left[\pi\left(r_{2}^{2}-r_{1}^{2}\right)+n w p\right]}{\pi\left(r_{2}+r_{1}\right)+n p}
$$

The formula of the heat transfer area is

$$
A_{0}=L \times\left(2 \pi r_{i}+2 \pi r_{o}+2 n p\right)
$$

This paper researches the effect of slit width $(w)$ and the number of slits $(N)$ on the distribution of flow field in the model with the same heat transfer area under various operating conditions. Fig. 2 shows the diagram of the series of slit models used in the simulation. When the influence of the slit width on the flow field distribution was investigated by simulation, the slit depth $p=5 \mathrm{~mm}$ and the number of slits $N=9$ were kept constant with varying slit width models $w=2.5 \mathrm{~mm}, 3.75 \mathrm{~mm}, 5 \mathrm{~mm}, 6.25 \mathrm{~mm}$, and $7.5 \mathrm{~mm}$. On the other hand, to determine the effect of the slit number $N$ on the distribution of the flow field, the slit width $w=7.5 \mathrm{~mm}$ was kept constant and the number of slits $N=6,9$, and 12 were varied and studied, respectively.

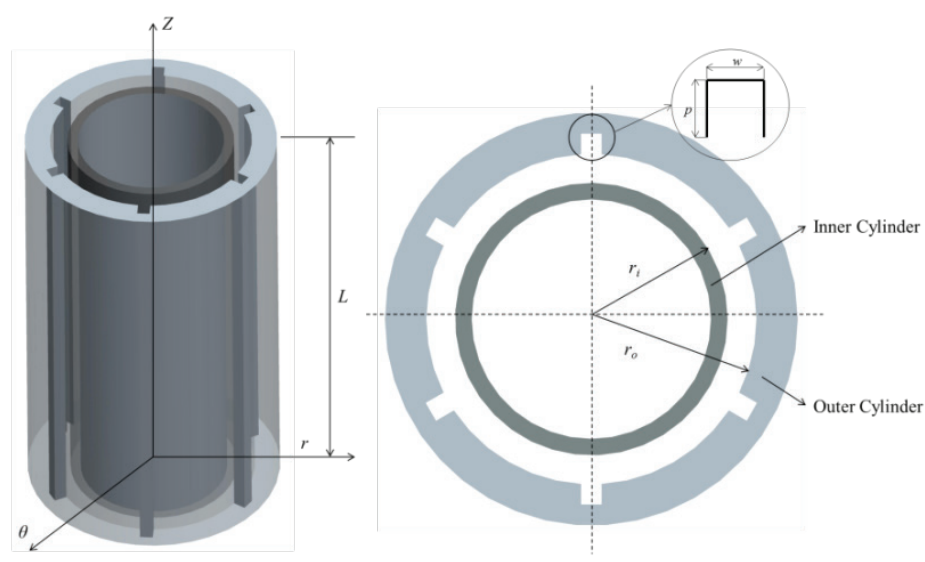

Figure 1. The slit model under investigation. 


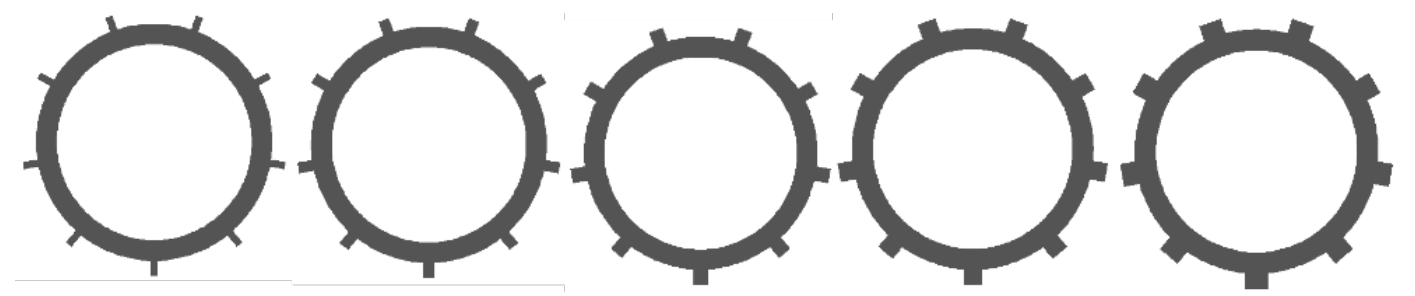

(a)

(b)

(c)

(d)

(e)

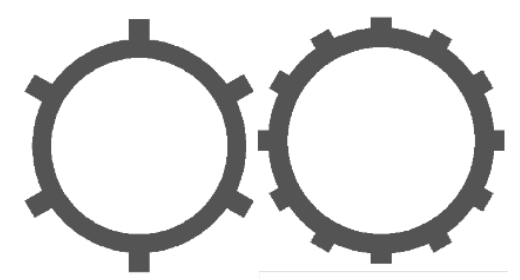

(f)

(g)

Figure 2. The diagram of the slit model (a) $\mathrm{w}=2.5 \mathrm{~mm}$; (b) $\mathrm{w}=3.75 \mathrm{~mm}$; (c) $\mathrm{w}=5 \mathrm{~mm}$; (d) $\mathrm{w}=6.25 \mathrm{~mm}$; (e) $\mathrm{w}=7.5 \mathrm{~mm}$; (f) $\mathrm{N}=6$; (g) $\mathrm{N}=12$.

\section{Numerical Methods and Boundary Condition}

Girimaji proposed a turbulence bridge model between the RANS model and DNS- PANS (Partially-Averaged Navier-Stokes method) to capture more flow field details when the flow regime is turbulent (Girimaji, 2006). The smooth transition of the turbulence model from the RANS method to the DNS method can be realized by changing two filtering parameters: the unresolved turbulent kinetic energy term $f_{k}$ and the unresolved turbulent dissipative term $f \varepsilon$. This paper modifies the standard $k-\varepsilon$ model as Partially-Averaged Naiver-Stokes method, and the standard $k-\varepsilon$ turbulence model is as follows.

The vortice viscosity equation is

$$
\mu_{t}=\rho C_{\mu} \frac{k^{2}}{\varepsilon}
$$

The turbulent kinetic energy equation is

$$
\left.\frac{\partial}{\partial t}(\rho k)+\frac{\partial}{\partial x_{i}}\left(\rho k u_{i}\right)=\frac{\partial}{\partial x_{j}}\left[\left(\mu+\frac{\mu_{t}}{\sigma_{k}}\right) \frac{\partial k}{\partial x_{j}}\right)\right]+G_{k}+G_{b}-\rho \varepsilon-Y_{M}+S_{k}
$$

The turbulent dissipative equation is

$$
\left.\frac{\partial}{\partial t}(\rho \varepsilon)+\frac{\partial}{\partial x_{i}}\left(\rho \varepsilon u_{i}\right)=\frac{\partial}{\partial x_{j}}\left[\left(\mu+\frac{\mu_{t}}{\sigma_{\varepsilon}}\right) \frac{\partial \varepsilon}{\partial x_{j}}\right)\right]+C_{1 \varepsilon} \frac{\varepsilon}{k}\left(G_{k}+C_{3 \varepsilon} G_{b}\right)+C_{2 \varepsilon} \rho \frac{\varepsilon^{2}}{k}+S_{\varepsilon}
$$


The variables used in the equations above are defined as follows:

$M t$ : vortice viscosity coefficient,

$\rho$ : density of the fluid,

$k$ : the turbulent kinetic energy term,

$\varepsilon$ : turbulent dissipative term,

$\mathrm{C} \mu, \mathrm{C}_{1 \varepsilon}, \mathrm{C}_{2 \varepsilon}$, and $\mathrm{C}_{3 \varepsilon}$; the empirical constant; $C_{\mu}$ is 0.09 ; $C_{1 \varepsilon}$ is $1.44, C_{2 \varepsilon}$ is 1.92 ; and $C_{3 \varepsilon}$ is 0.99 ,

$G_{k}$ : turbulent kinetic energy generation term due to the time-averaged velocity gradient,

$G_{b}$ : floatinglLift-induced turbulent kinetic energy generation,

$S_{k}$ and $S \varepsilon$ : source term,

$\sigma_{k}-k$ : corresponding Prandtl number ; $\sigma_{k}$ for 1.0

$\sigma_{\varepsilon}-\varepsilon$ : corresponding Prandtl number ; $\sigma_{\varepsilon}$ for 1.3 .

With standard $k-\varepsilon$ compared with the model and PANS model compared mainly to coefficient $C_{2 \varepsilon}$ and Prandtl number $\sigma_{k}, \sigma_{\varepsilon}$ revises

$$
\begin{gathered}
C_{2 \varepsilon}{ }^{*}=C_{1 \varepsilon}+\frac{f_{k}}{f_{\varepsilon}}\left(C_{2 \varepsilon}-C_{1 \varepsilon}\right) \\
\sigma_{k u}=\sigma_{k} \frac{f_{k}^{2}}{f_{\varepsilon}}, \sigma_{\varepsilon u}=\sigma_{\varepsilon} \frac{f_{k}^{2}}{f_{\varepsilon}}
\end{gathered}
$$

The model is classified as a RANS model when $f_{k}=f_{\varepsilon}=1.0$. However, as $f_{k}$ decreases gradually, the model transients to the DNS model. In this paper, more changes in the flow in the annular gap were captured by PANS model which was modified by Software Fluent. PANS model was built for this study based on fluent software for more subtle flow field changes. The rotating axis of the inner cylinder is the $Z$-axis with a stationary outer cylinder. The radial temperature gradient is considered in this paper. Therefore, the upper and lower walls are set as static insulation surfaces. The temperature of the outer cylinder and the ambient is $24^{\circ} \mathrm{C}$ while the temperature and speed of the inner cylinder, on the other hand, depend on the working condition.

The flow field distribution and heat transfer of two concentric cylinder annuluses were numerically simulated by the commercial software ANSYS Fluent15.0. The turbulent Taylor-Couette flow was numerically simulated by using a pressure-based semi-implicit solver to obtain the coupling equations of pressure and velocity. SIMPLEC algorithm was adopted for the solution method and the second-order upwind scheme for the pressure, turbulent kinetic energy, and the turbulence dissipation. QUICK format, on the other hand, was used for the convective terms.

\section{VALIDATION OF NUMERICAL SIMULATION}

Tachibana's experimental model (Tachibana, 1960) was used in validating the applied PANS model in the simulation which is shown in Fig. 3. The radii of the inner and outer cylinders are fixed at $120 \mathrm{~mm}$ and $160 \mathrm{~mm}$, respectively, with an axial dimension of $210 \mathrm{~mm}$. The temperature difference between the inner and outer cylinders is $20^{\circ} \mathrm{C}$ with a working fluid of water. The empirical equation of the averaged Nusselt number is $\mathrm{Nu}=0.42\left(\mathrm{Ta}^{2} \mathrm{Pr}\right)^{1 / 4}$. The formulas of Nusselt number and Taylor number at the inner cylinder are 


$$
\begin{aligned}
& N u=\frac{2 h d}{\lambda}=\frac{2 Q d}{\left(T_{\text {in }}-T_{\text {out }}\right) A \lambda}=\frac{2 q d}{\Delta T \lambda} \\
& T a=\frac{v_{t} d}{v} \sqrt{\frac{d}{r_{i}}}
\end{aligned}
$$

In the formula, $h$ is the overall heat transfer coefficient; $h=Q / \Delta T \lambda ; d$ is the annular gap width; $A$ is the inner wall surface area; $\lambda$ is the thermal conductivity of the fluid; $\Delta T$ is the temperature difference between the inner and outer cylinder surfaces, $\Delta T=T_{\text {in }}-T_{o u} t ; q$ is Wall heat flux; $v$ is the dynamic viscosity; $v_{t}$ is the circumferential speed of the inner cylinder, and $r_{i}$ is the radius of the inner cylinder.

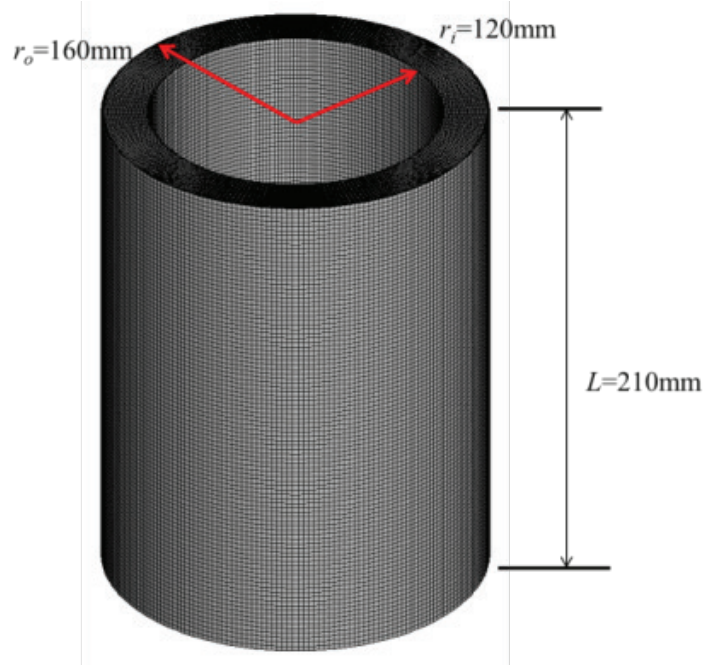

Figure 3. Simulation mesh model.

The distribution of the averaged Nusselt number at the inner cylinder was observed with varying inner cylinder rotating speeds: $100 \mathrm{rpm}, 200 \mathrm{rpm}, 300 \mathrm{rpm}, 400 \mathrm{rpm}$, and 500rpm. The simulation results were compared with the experimental results extracted from Tachibana's study (Tachibana, 1960). It can be understood from Fig. 4 that when $f_{k}$ decreases gradually, the numerical simulation results and the experimental solutions are slightly close. Hence, the effect of $f_{k}$ on the heat transfer process gradually becomes larger when the rotating speed of the inner cylinder increases.

When the rotation speeds are 100rpm, 200rpm, 300rpm, 400rpm, and 500rpm, the difference between the numerical simulation results and the experimental results of the $f_{k}=0.3$ PANS model is $4.068 \%, 0.815 \%, 3.147 \%$, $3.385 \%$, and $4.498 \%$, respectively. The simulation results are nearly consistent with the experimental results. The accuracy of the numerical simulation was thus confirmed since both the numerical simulation and experimental results of heat transfer performance in the annulus were found to be in good agreement. The validated PANS method would then be applied in the heat flow characteristics study in the different slit wall models. 


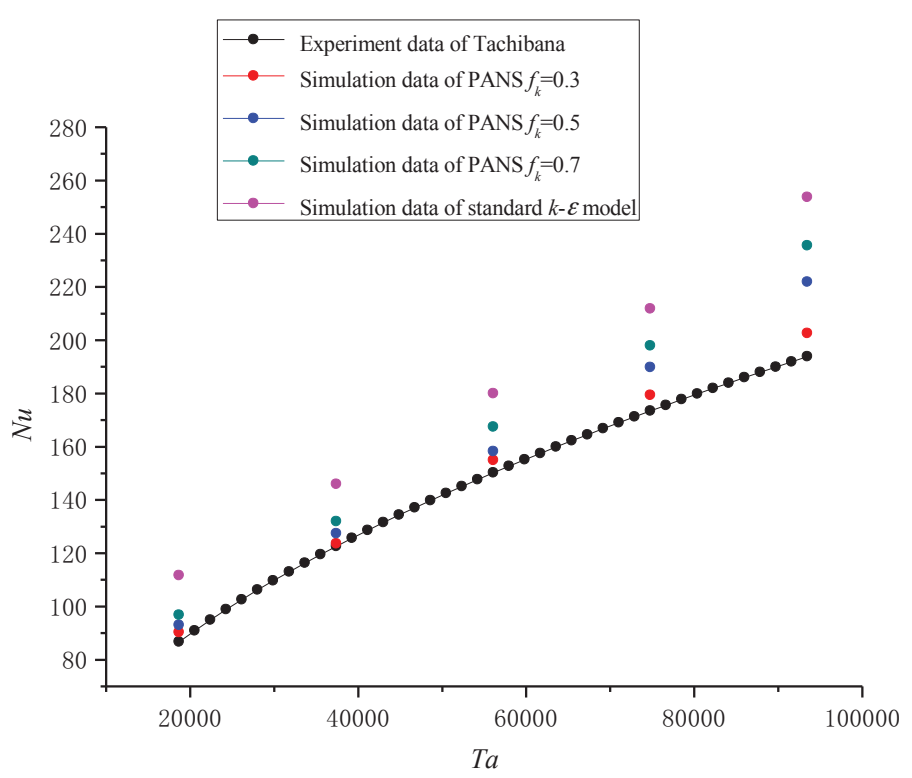

Figure 4. Distribution of the averaged Nusselt number at the inner cylinder with different rotating speeds.

\section{RESULTS AND DISCUSSION}

Numerical simulations of fluid flow and heat transport in Taylor-Couette model are conducted in this work with the following variety of parameters:

working fluid: water with constant properties, Reynolds number (Re): $2000 \leq \mathrm{Re} \leq 5000$, slit width (w): $2.5,3.75,5,6.25$, and $7.5 \mathrm{~mm}$, number of slits $(\mathrm{N}): 6,9$, and 12 .

Results and discussion recorded here highlight the influences of width and numbers of slits at different values of Reynolds numbers on thermal characteristics behavior and flow field, with temperature gradient effects taken into account and without it.

\section{MEAN ISOTHERMAL FLOW CHARACTERISTICS}

Figure 5 graphically presents the two-dimensional velocity field distribution with different slit width models under the isothermal condition, through constant magnitude of Reynolds number $\left(R e_{\omega}=2000\right)$, under isothermal condition. It was observed that Taylor vortex gradually deforms as the slit width increases. The influence of the fluid in the mainstream area in the slit gradually becomes larger and the entire flow field tends to be disordered. To explain this phenomenon, the $R-Z$ plane annular middle $\left(R^{*}=0.5\right)$ of the radial velocity distribution in Fig. 6 was extracted. The change in the slit width is noted to have an obvious influence on the radial velocity distribution. Also, the radial velocity extreme value increases with an increase in the width of the slit resulting in a higher absolute value of positive radial velocity than the reverse. The absolute value of the radial velocity is largely related to the slit width increase. Subsequently, it is observed that the jet wall strength between the inner and outer walls increases and becomes stronger than the reverse. 


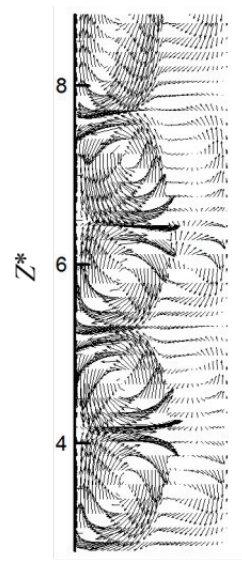

(a)

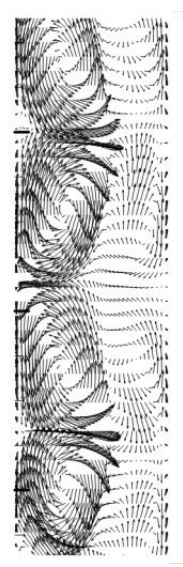

(b)

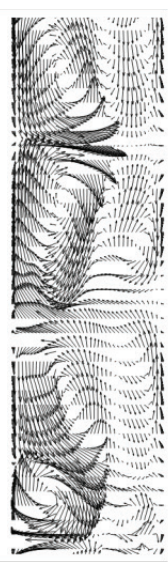

(c)

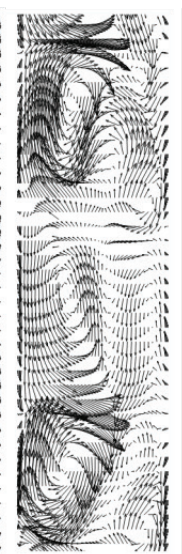

(d)

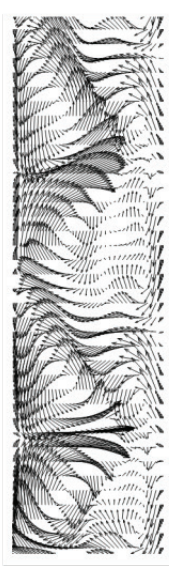

(e)

Figure 5. Velocity distribution of different slit width models in the $R-Z$ plane (a) $\mathrm{w}=2.5 \mathrm{~mm}$; (b) $\mathrm{w}=3.75 \mathrm{~mm}$; (c) $\mathrm{w}=5.0 \mathrm{~mm}$; (d) $\mathrm{w}=6.25 \mathrm{~mm}$; (e) $\mathrm{w}=7.5 \mathrm{~mm}$.

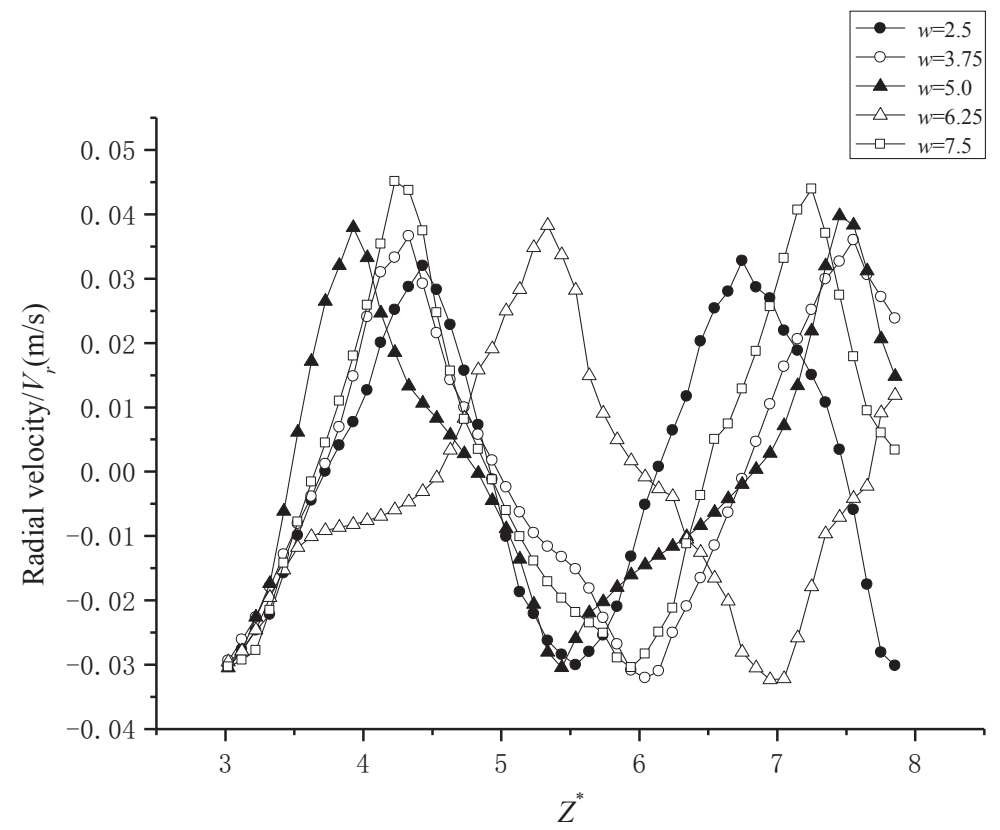

Figure 6. Radial velocity distribution of different slit width models along the axial direction.

The distribution of wall shear stress, turbulence intensity, and radial velocity $\left(R^{*}=0.5\right)$ near the inner cylinder surface $\left(R^{*}=0\right)$ of the $w=2.5 \mathrm{~mm}$ model are shown in Fig. 7. The velocity periodically changes along the axial direction with the maximum radial velocity of the forward direction being greater than the minimum radial velocity of the opposite direction. This indicates that turbulent Taylor-Couette flow is the product of the periodic action of the strong out wall jet and the weak inflow jet. Also, the turbulence intensity and the wall shear stress at the inner cylinder surface are at the minimum in the position of the maximum radial velocity. This is because the area with the largest radial velocity exhibits the strongest outflow jet intensity. Hence, there is a tendency to break away from the wall surface when the fluid near the inner cylinder is affected by the out wall jet flow. This will cause the wall shear stress 
exerted on the fluid in the wall surface to become smaller. The kinetic energy of the fluid in the annulus can as well be associated with the rotating inner cylinder. When the fluid near the wall surface breaks away from the cylinder, the interaction between the cylinder and the fluid lessens. Also, the kinetic energy transmitted from the inner cylinder to the fluid near the wall is smaller which implies a smaller turbulent intensity at this position. It can be concluded that the rotating cylinder exerts a wall shear stress on the fluid near the cylinder and also transmits kinetic energy to the fluid in the annulus through the wall shear stress.

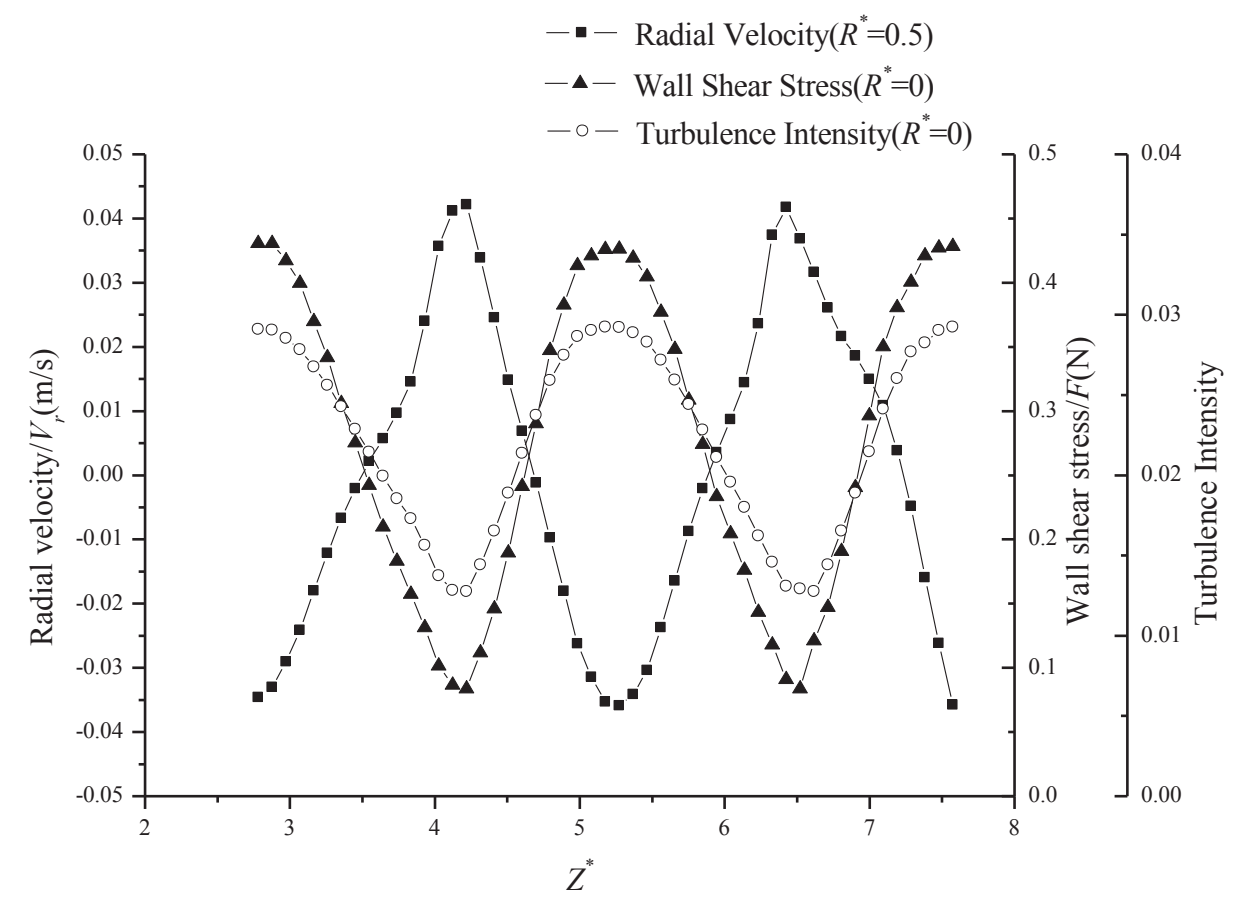

Figure 7. Distribution of the wall shear stress, turbulence intensity, and radial velocity along the axial direction $\left(R e_{\omega}=2000\right)$.

Figure 8 shows the radial velocity at the middle position of the annulus, the turbulence intensity of the fluid near the inner cylinder, and the maximum value of the wall shear stress of the five values of Reynolds number $\left(R e_{\omega}=2000,2884,3768\right.$, and 4682), respectively. The result shows that with an increase in Reynolds number, the radial velocity, turbulence intensity, and wall shear stress will increase. Thus, as Reynolds number increases, the difference between the individual models gradually increases too. This indicates that when Reynolds number increases, the intensity of jet flow between walls becomes higher. Turbulent Taylor vortex is noted to be the mode through which the jet kinetic energy is dissipated from a rotating inner wall into the mainstream. Therefore, an increase of Reynolds number will enhance the jet vortex, promote the radial movement of fluid in annular, and lead to the increase of turbulence intensity in the middle of the ring gap position as well. Similarly, the fluid and wall friction near the inner wall intensify, causing an increase in the wall shear force near the liquid. The result shows that when Reynolds number increases, the convection intensity between the inner and outer walls becomes stronger. Furthermore, an increase of the slit width strengthens the effect of the vortex on the jet flow between the inner and outer walls, leading to a gradual impact on the distribution of the convection field. 


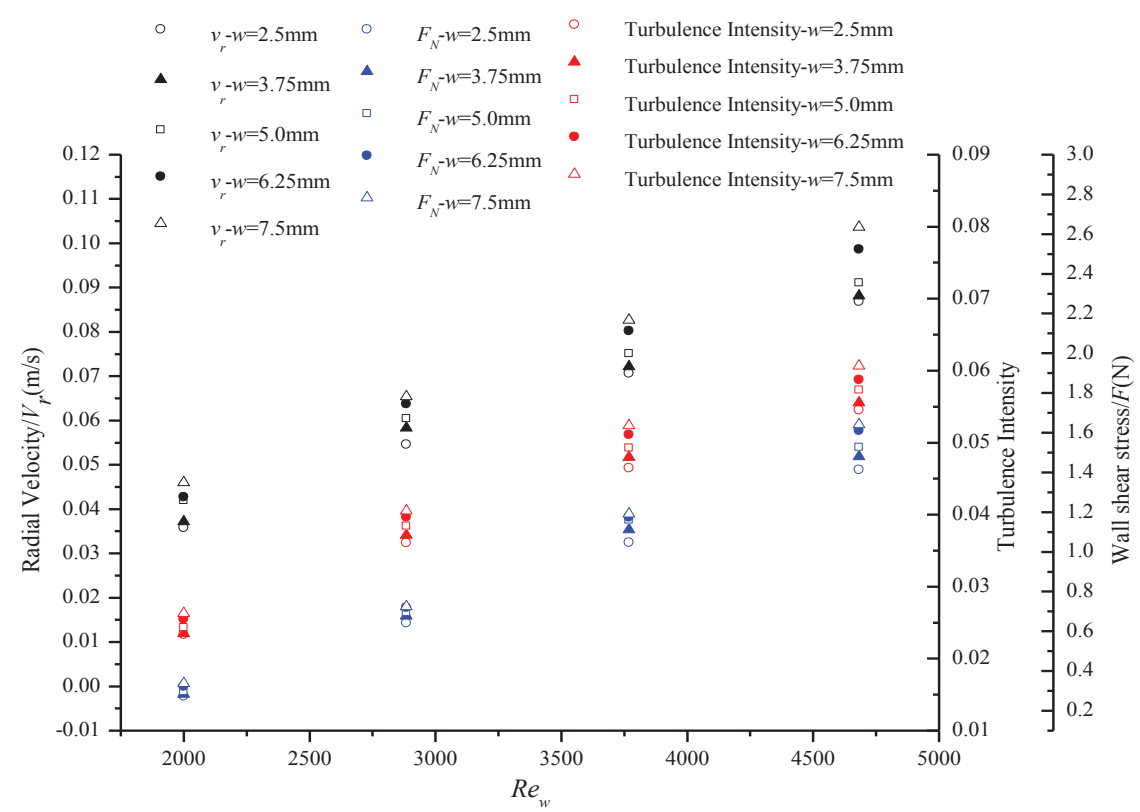

Figure 8. Distribution of radial velocity, shear force, and turbulence intensity at different Reynolds numbers.

\section{SILT NUMBER EFFECT ON ISOTHERMAL FLOW}

Figure 9 shows the velocity vector distribution of the model with the slit width $\mathrm{w}$ of $7.5 \mathrm{~mm}$ and the number of slits 6, 9, and 12, respectively, when $R e_{\omega}=2000$. As shown in the figure, when the number of slits increases, both the axial size of Taylor vortex in the mainstream area and the near-wall small-scale vortex decrease. Since the wet periods of the three models are the same, an increase in the number of slits results in a gradual decrease in the slit depth $p$. The small-scale vortex of the slit area will then have a greater effect on Taylor vortex in the mainstream area causing slight turbulence in Taylor vortex.

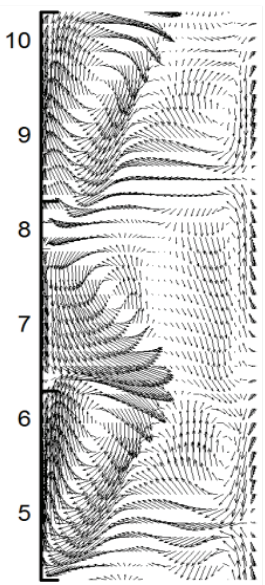

(a) $N=6$

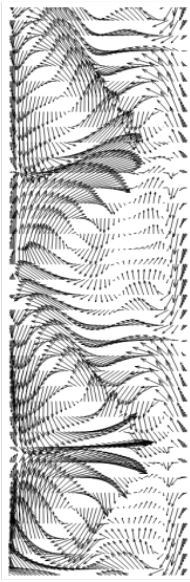

(b) $N=9$

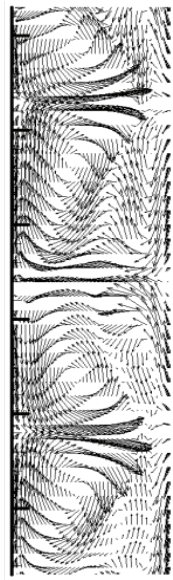

(c) $N=12$

Figure 9. Distribution of velocity vectors with different slit number $\left(R e_{\omega}=2000\right)$. 


\section{MEAN NON-ISOTHERMAL FLOW CHARACTERISTICS}

Figure 10 shows the velocity vector distribution of the $R-Z$ plane for different slit width models with $R e_{\omega}=2000$. When the width w of the slit is $2.5 \mathrm{~mm}$, Taylor vortex shape in the annulus is more regular. But when the width of the slit increases, Taylor vortex gradually deforms, and the result shows a similarity to the isothermal condition. Also, when the width of the slit increases, the flow field tends to be disordered.

To analyze the effect of slit width on the distribution of the annular flow field, the vorticity distributions of the five model $R-Z$ planes are extracted. As shown in Figure 11, the slit width has a significant influence on the vorticity distribution. When the slit width gradually increases, the annulus flow field gradually shifts from a relatively stable flow field to a turbulent flow field. Also, when the width of the slit increases, the small-scale vortex strength of the slit area rises significantly. The area affected by it thus becomes larger, resulting in a more fluid exchange between the mainstream area and the slit area. The main vortex and the small-scale vortex are noted to directly affect each other. Hence, in Taylor vortex pair, the size difference between two adjacent vortexes becomes larger with a disordered vortex shape as well.

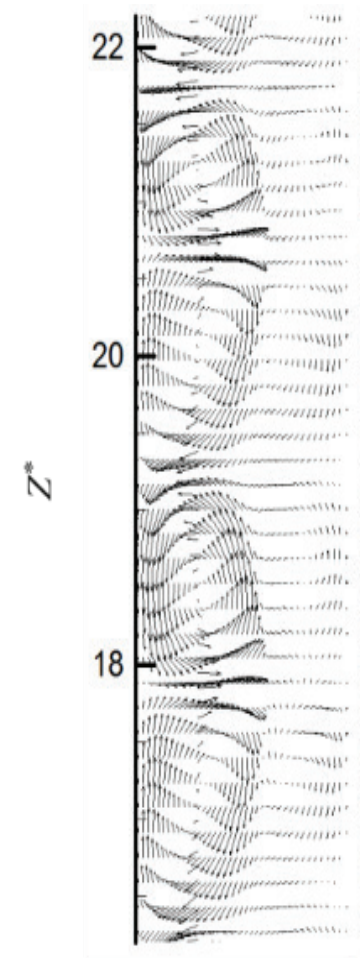

(a)

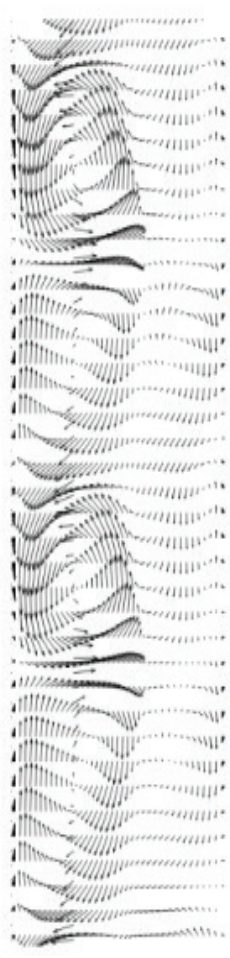

(b)

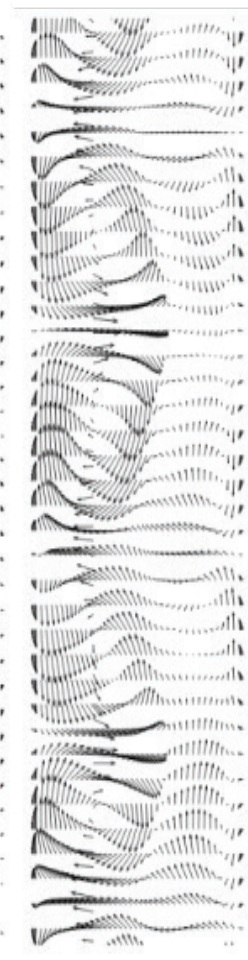

(c)

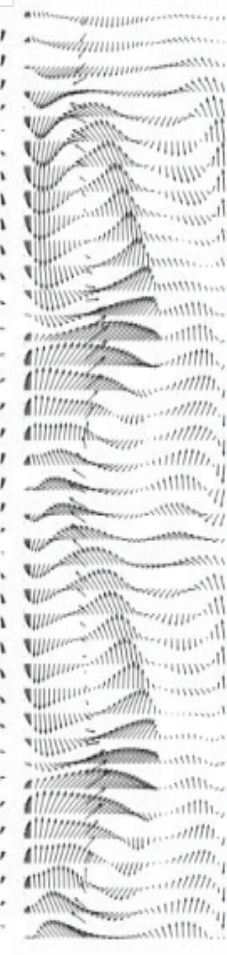

(d)

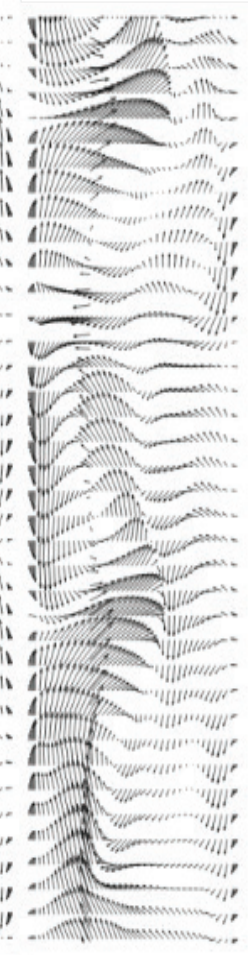

(e)

Figure 10. $R-Z$ plane velocity distribution for different slit width models.

(a) $w=2.5 \mathrm{~mm}$; (b) $w=3.75 \mathrm{~mm}$; (c) $w=5.0 \mathrm{~mm}$;(d) $w=6.25 \mathrm{~mm}$; (e) $w=7.5 \mathrm{~mm}$. 


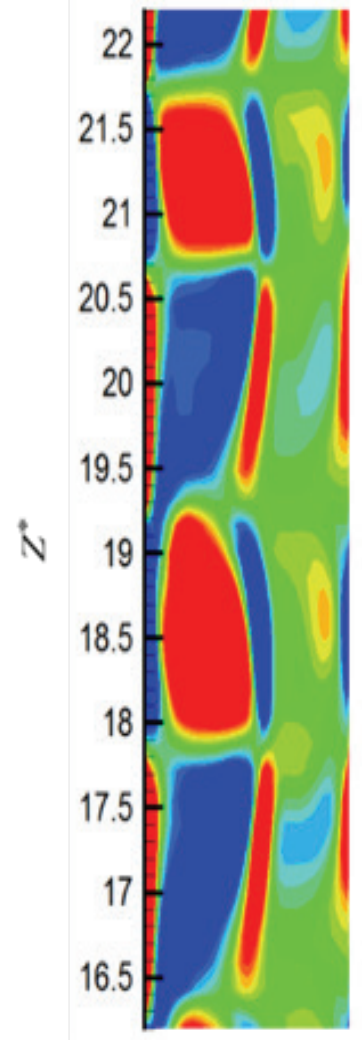

(a)

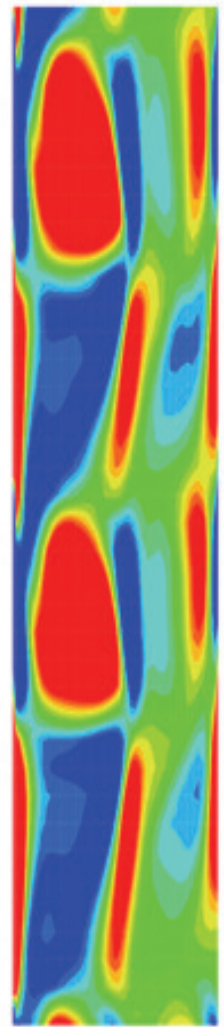

(b)



(c)

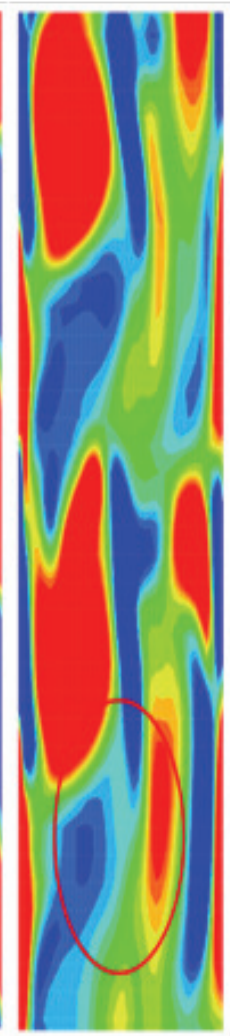

(d)

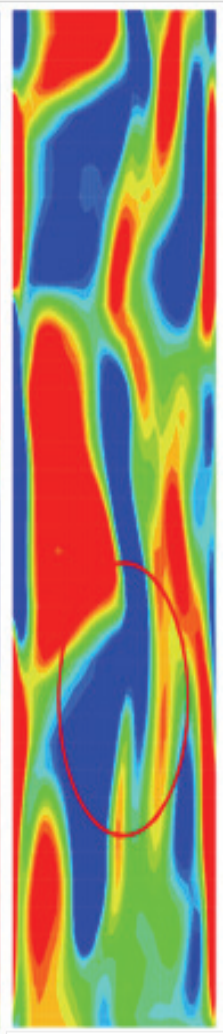

(e)

Figure 11. $R$ - $Z$ plane vorticity distribution in different slit width models.

(a) $w=2.5 \mathrm{~mm}$; (b) $w=3.75 \mathrm{~mm}$; (c) $w=5.0 \mathrm{~mm}$; (d) $w=6.25 \mathrm{~mm}$; (e) $w=7.5 \mathrm{~mm}$.

\section{VELOCITY FIELD AND HEAT TRANSFER İN THE SLIT AREA}

Figure 12 shows the velocity and temperature distribution of Taylor vortex in the middle $\left(Z_{3}\right)$ slit area. Generally, the two extreme regions of temperature are consistent with the two extreme regions of velocity. This indicates that, on the left side of the slit, the fluid in the mainstream hits the wall and forms a high-velocity region, resulting in a better mixing and heat exchange between the hot and cold fluids. The velocity of the fluid in the slit is gradually reduced by the viscous force between the fluid and the friction force of the wall along the rotation direction of the vortex thus forming a low-velocity area on the right wall of the slit. Fluid velocity in the region is low and the interfluid mixing effect is also weakened due to the mixing and heat exchange between the fluids before. As a result, the temperature of the hot fluid gradually decreases and approaches to the temperature of the cold fluid. The temperature difference between the fluid and the outer wall surface becomes smaller causing the heat exchange to be weak bringing about a low-temperature zone at the location. It is noted that the distribution of the velocity field and temperature field in the slit area is the same. In conclusion, the mixing effect and the heat exchange between the hot and cold fluid on the left area of the slit are more severe. This is because the temperature difference and the fluid flow velocity between the fluid and the wall are high. 


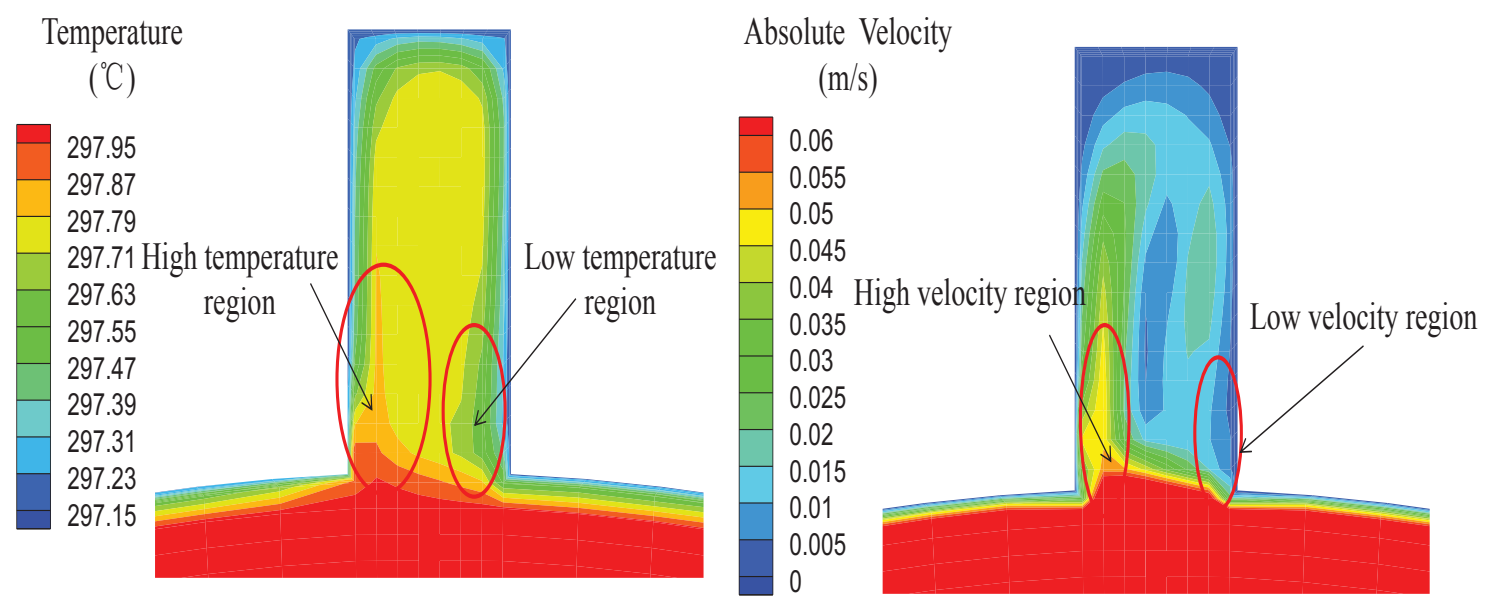

Figure 12. Distribution of temperature and velocity fields in the silt area

$$
\left(R e_{\omega}=2000 ; w=2.5 \mathrm{~mm}\right) \text {. }
$$

To further analyze the effect of slit width on the formation of small-scale vortices and their strength, the velocity vector and velocity distribution of Taylor's vortex center position $\left(Z_{3}\right)$ were extracted. As shown in Fig. 13, when the fluid in the mainstream region passes through the slit region, it is affected by the inertial force and boundary layer detachment. This causes the fluid to hit the left wall of the slit to form a small-scale vortex which is consistent with the results under isothermal conditions. Under the influence of the viscous force between the fluids and the shear force generated by the relative motion of the fluid and the wall surface, the fluid velocity gradually decreases along the swirling direction of the vortex. and a low-velocity region appears. The small-scale vortex energy in the slit area promotes the fluid exchange between the mainstream area and the slit area. Therefore, when the area with lowvelocity value is large, the exchange effect between the mainstream and the fluid in the slit becomes weak.

According to the conclusion in Figure 12, with a higher speed within the slit area, the mixing effect between the hot and cold fluids and the heat exchange are more severe when compared with the distribution of the velocity field of each model in Figure 13. It is found that when the slit width increases, the area with low velocity decreases significantly which is an indication that the heat transfer effect of the fluid in the slit area of the model $w=7.5 \mathrm{~mm}$ is the best.

\section{SLIT WALL EFFECT ON WALL SHEAR STRESS AND HEAT FLUX}

In order to further explain the phenomenon that the velocity of the $\mathrm{w}=7.5 \mathrm{~mm}$ model is significantly higher in the slit area, the distribution of shear forces on the wall 0-1 wall surface of the slit of different models is extracted, as shown in Figure 14. The shear force initially shows an increasing trend and later decreases rapidly after reaching the maximum value. At the same time, when the width of the slit increases, the shear force exerted on the fluid in the wall area gradually increases. When extreme point appears at a deeper position in the slit, the slit depth $p$ is larger. With the increase of the width of the slit, the increasing trend of the shear force gradually becomes smaller. According to the previous analysis, the fluid in the mainstream region hits the left side of the slit to form a small-scale vortex under the influence of the inertial force and the delamination of the boundary layer which shows an increasing trend in shear force. The largest shear force is the point that the mainstream impacts the slit wall. Due to the influence of both frictions between the fluid and the wall and the fluid, the velocity gradually decreases along the rotation direction of the vortex. The results show that the fluid has a tendency to flow to the slit while continuing to maintain the circumferential movement due to the inertia force and the detachment of the boundary layer. 


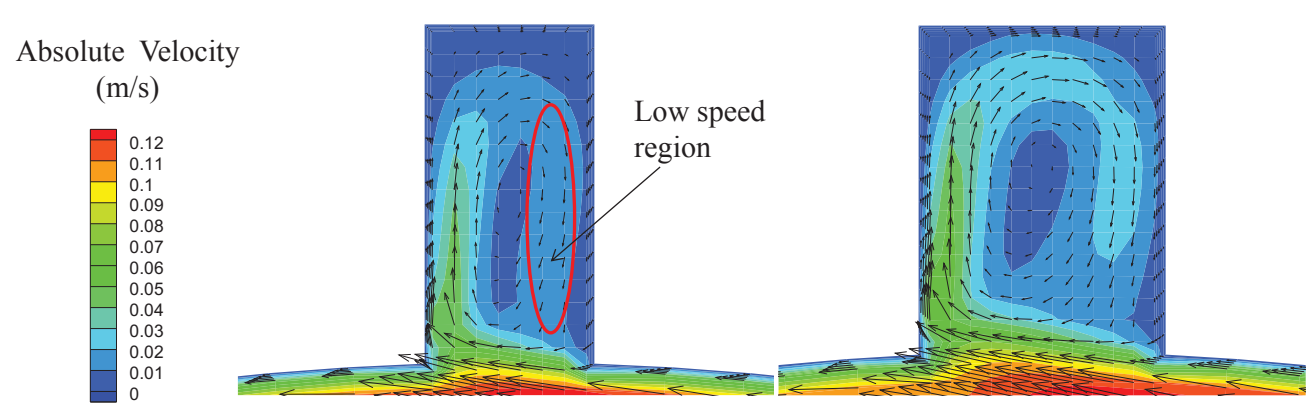

(a)

(b)

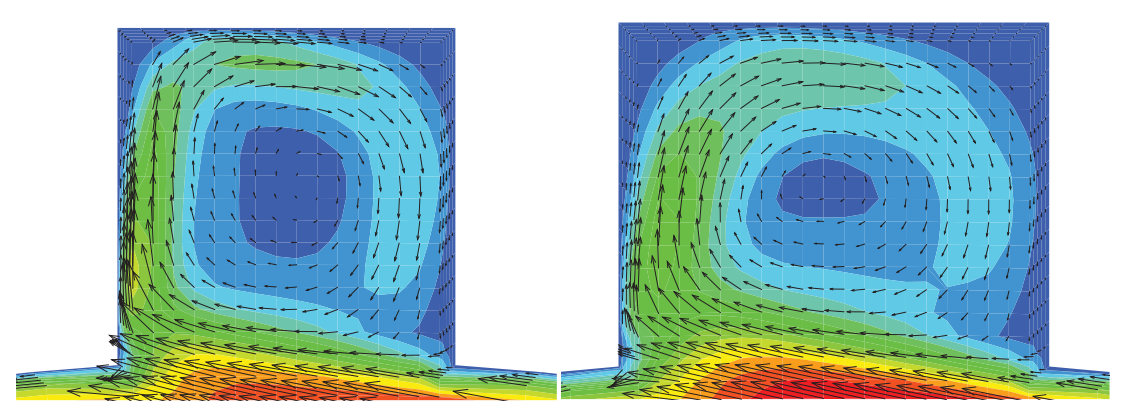

(c)

(d)

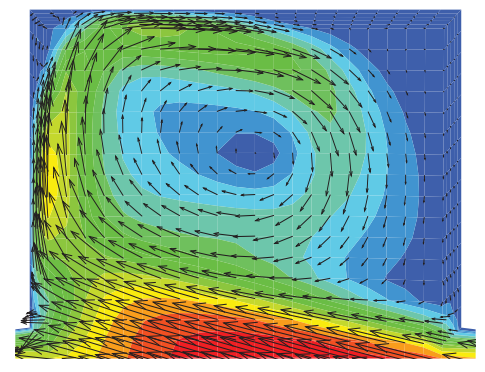

(e)

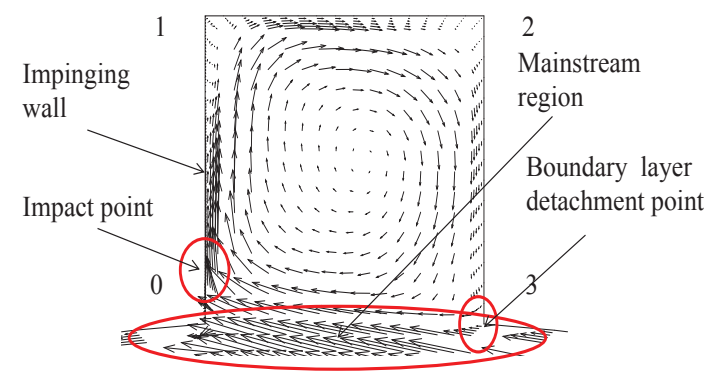

(f)

Figure 13. Distribution of the velocity field in the slit area (a) $w=2.5 \mathrm{~mm}$; (b) $\mathrm{w}=3.75 \mathrm{~mm}$; (c) $\mathrm{w}=5.0 \mathrm{~mm}$; (d) $\mathrm{w}=6.25 \mathrm{~mm}$; (e) $\mathrm{w}=7.5 \mathrm{~mm}$; (f) slit flow field diagram.

When the width of the slit increases, this kind of trend has a greater influence on the fluid. The fluid impacts the slit in a deeper point, resulting in less velocity loss and greater fluid velocity of the fluid in the slit area. The increase of the wall shear force with the increase of the slit width is because when the width of the slit increases, the highspeed fluid in the mainstream area has a greater influence on the slit area, resulting in fluid striking the wall at a greater velocity. Since the mainstream fluid of the model with a larger slit width hits the wall surface with greater velocity, the impact point is deeper. The slit width is larger and the fluid in the slit area of the model is exchanged more violently with the mainstream fluid resulting in a stronger fluid mixing effect and heat exchange. Generally, the $w=7.5 \mathrm{~mm}$ model has the optimal heat transfer effect among the five models. 
Figure15 shows the distribution of radial velocity, inner wall shear force, and maximum heat flux density for different slit width models at rotational Reynolds number $R e_{\omega}$ of $2000,2884,3768$, and 4682, respectively. When Reynolds number increases, the radial velocity in the middle of the annulus, the shearing force of the inner wall surface, and the maximum heat flux density tend to increase. Consequently, when Reynolds number increases, the difference between the three quantities gradually becomes larger. Similarly, at different Reynolds numbers, the three physical quantities increase as the width of the slit increases since the maximum value of radial velocity and heat flux is the strongest at the region of the outer jet. The radial motion of the fluid also promotes thermal convection between the inner and outer walls. The stronger the convection force between the inner and outer walls, the greater the shear force between the fluid and the wall, and the better the heat transfer effect of the model.

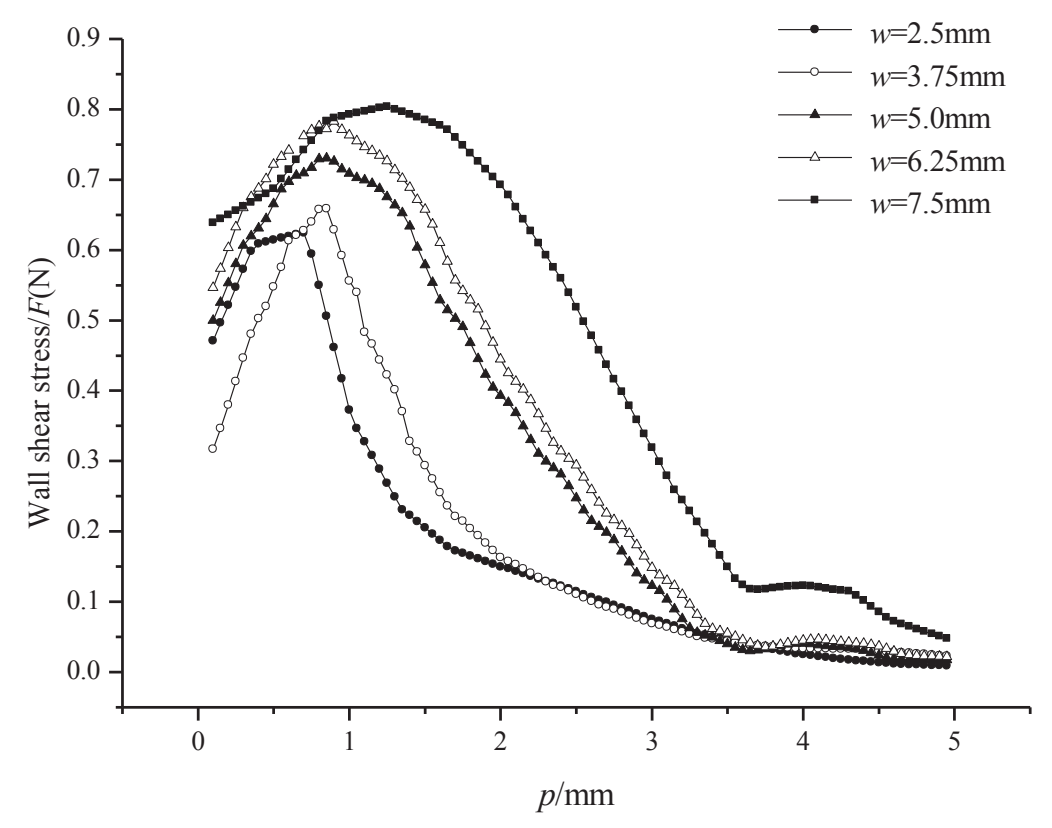

Figure 14. Distribution of shear force on wall surface impacted by slit width $\left(R e_{\omega}=2000 ; \Delta T=2^{\circ} \mathrm{C}\right)$.

In summary, the increase in the rotational speed of the inner wall surface will make the jet action between the inner and outer wall surfaces stronger, resulting in a stronger convection strength of the model and an enhanced heat transfer effect of the model. Also, the heat transfer capacity of the model gradually becomes stronger at a certain range with an increase in the width of the slit.

\section{SLIT NUMBER EFFECT ON WALL SHEAR STRESS AND HEAT FLUX}

To further study the effect of the number of slits on the external wall shear force, the wall shear force distributions of the three models $(N=6,9$, and 12 slit) hitting the wall surface were examined. As shown in Figure 16 , when the number of slits increases, the position of the impact point on the impact wall surface becomes shallow, and the extreme value of the wall surface shear force becomes large. This is because when the number of slits increases, the small-scale vortices in the slit region are pressed in the radial direction, causing the collision point to gradually advance. 


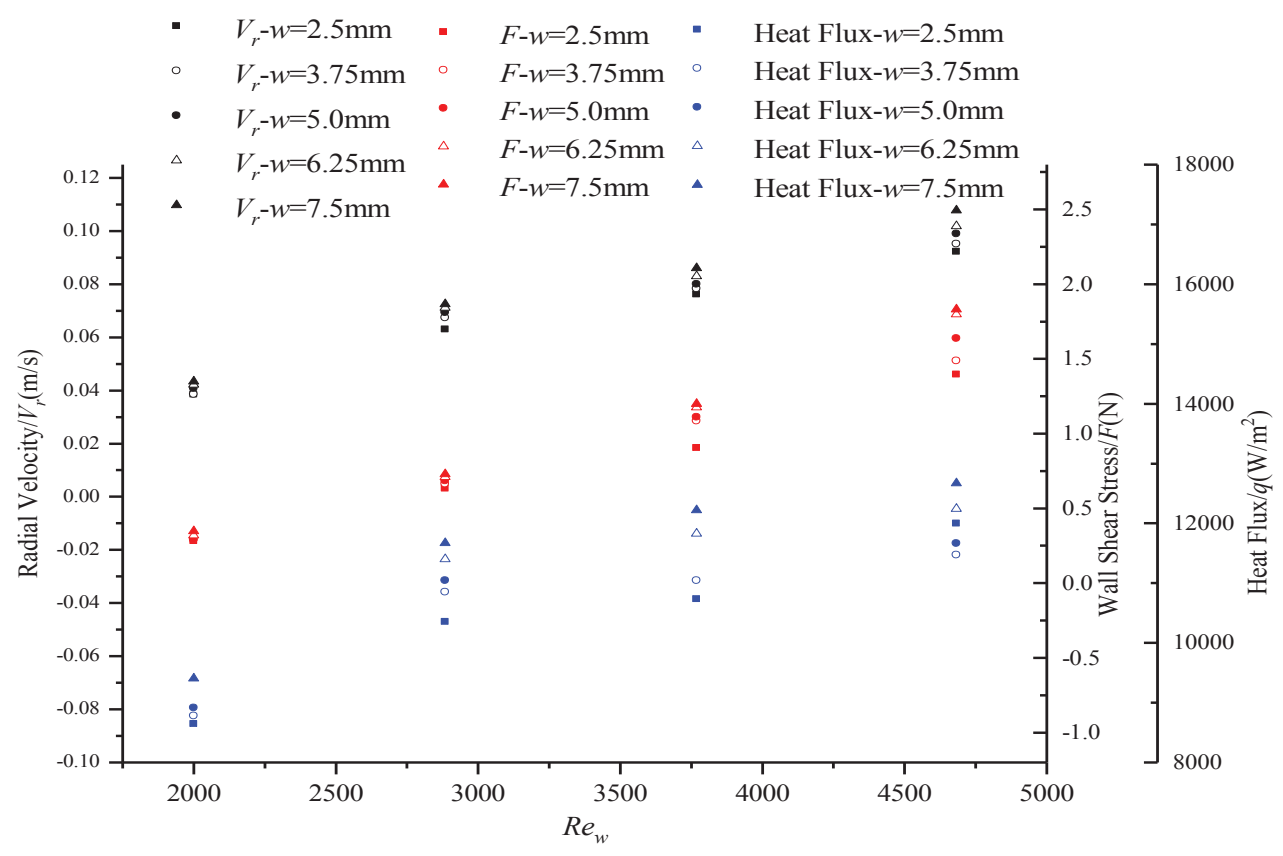

Figure 15. Distribution of physical quantities at different Reynolds numbers.

When the impact point moves forward, the distance where the fluid in the mainstream region moves to the position of the impact point becomes shorter causing the velocity loss of the fluid to be smaller. Therefore, the shear force exerted by the fluid on the wall surface becomes large. The depth of the slit also becomes shallow as a result of the fluid loss due to the viscous force and the friction force decrease after the fluid moves against the wall surface. The higher the speed of the slit area, the better the heat transfer capacity of the model. Therefore, increasing the number of slits within a certain range can enhance the heat transfer performance of the model.

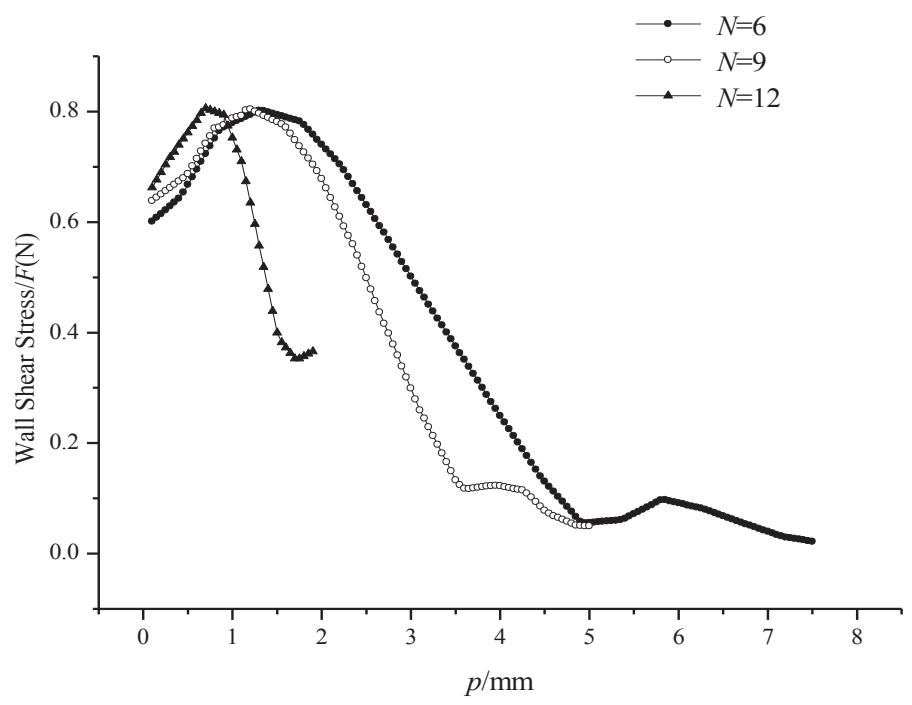

Figure 16. Distribution of wall shear force of different slit number models $\left(R e_{\omega}=2000 ; \Delta T=2^{\circ} \mathrm{C}\right)$. 


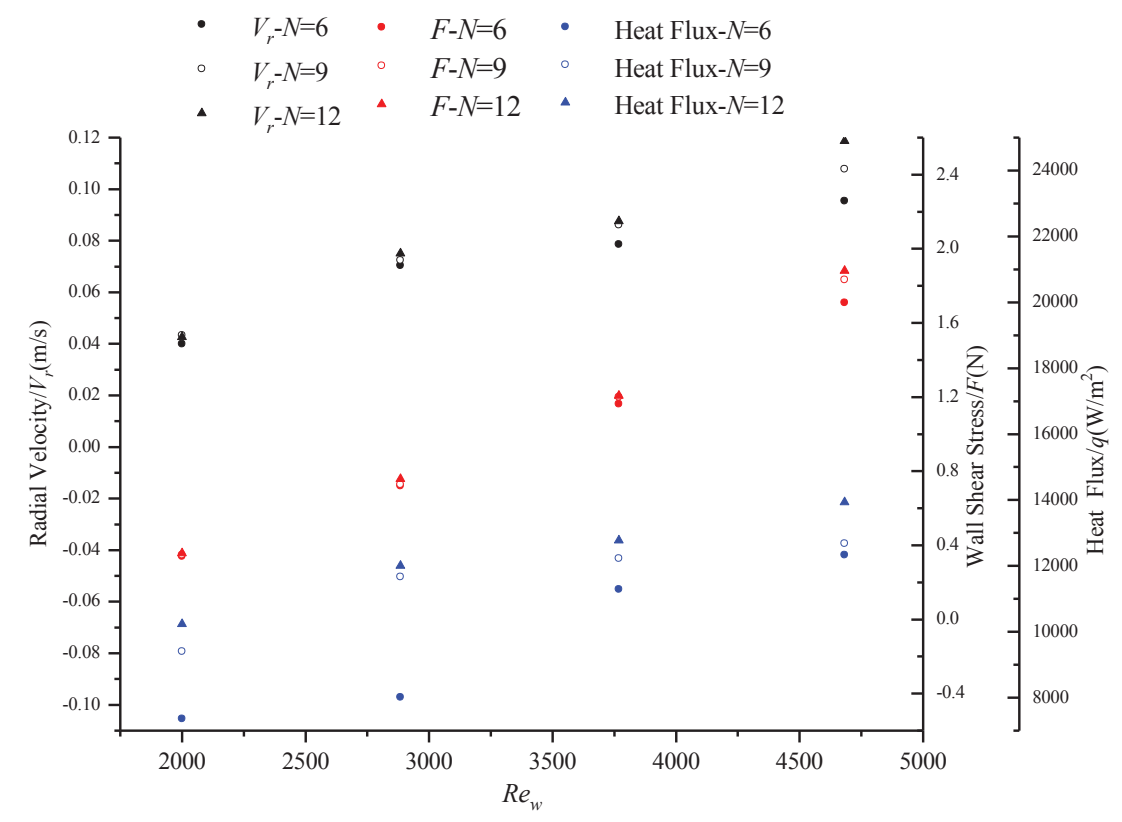

Figure 17. Distribution of physical quantities in different slit number models.

Figure17 shows the physical quantity distribution of each slit number model at different Reynolds numbers. When the number of slits increases, the radial velocity, the internal wall surface shear force, and the maximum value of heat flux increase too. Also, when Reynolds number increases, the difference between the physical quantities increases. The increase in the number of slits only changes the location of the impact point and the fluid loss distance. Compared with the fluid loss caused by friction and viscous forces, the influence of mainstream fluid on the fluid in the slit is more obvious.

\section{AVERAGE NUSSELT NUMBER}

To further study the heat transfer performance of each model, the average Nusselt number $(\mathrm{Nu})$ distribution on the inner wall surface is obtained when different Reynolds numbers are extracted. The average Nusselt number on the inner wall surface of the slit model is defined as

$$
\overline{N u}=\frac{\bar{q} \cdot 2 D_{h}}{\Delta T \cdot \lambda}
$$

$q$ is the average heat flux of the inner wall surface; $D_{h}$ is the equivalent diameter of the model; $\Delta T$ is the temperature difference between the inner and outer walls; and $\lambda$ is the thermal conductivity of the annulus fluid. As shown in Figure 18, Reynolds number is directly proportional to Nusselt number of different models. The results show that the heat transfer capacity of the model increases when Reynolds number increases. Also, the effect of the slit width on the heat transfer capacity of the model gradually becomes larger. The model of slit width is $7.5 \mathrm{~mm}$ and it is noted to have the best heat transfer performance among the five models.

Figure19 shows the average Nusselt number distribution on the inner wall surface of each slit model at different Reynolds numbers. As shown in the figure, an increase in the number of slits is directly related to the average Nusselt 
number which causes the heat transfer performance of the model to increase too. Figure 19 is noted to be similar to Figure 18. As the difference in Nusselt number of different slit number models increases, the Reynolds number increases as well. The result shows that the increase in the number of slits within a certain range can improve the heat transfer performance of the model. Reynolds number has less effect on the heat transfer performance of the model at a higher number. Moreover, the heat transfer performance of the model becomes greater due to the influence of the slit wall geometry.

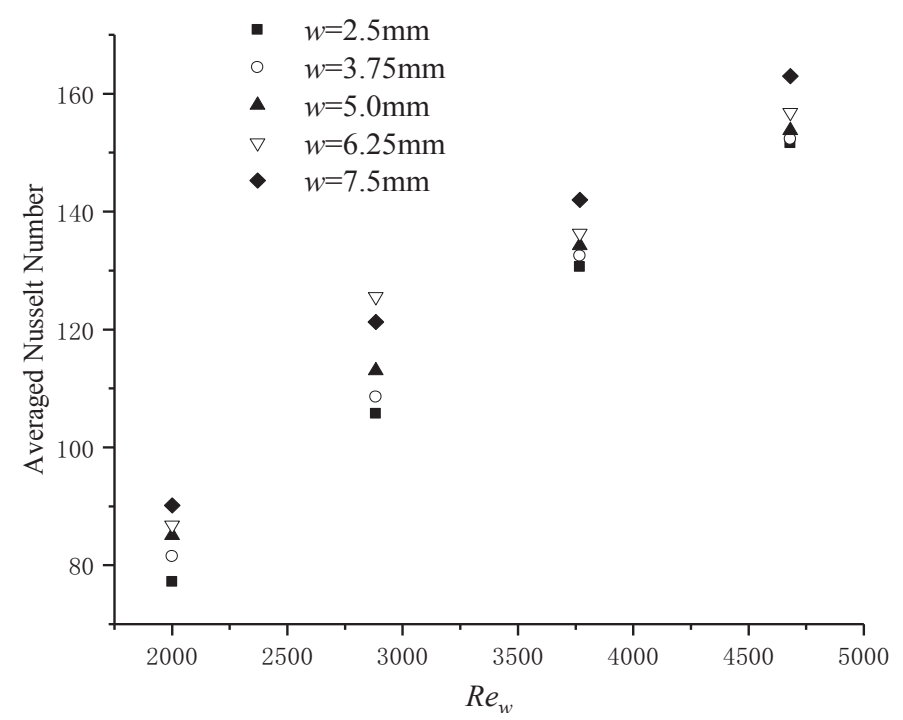

Figure 18. Nusselt number distribution in different slit width models.



Figure 19. Average Nusselt number distribution on the inner wall surface of the different number of silts. 


\section{CONCLUSION}

The influence of slit number and width on the distribution of flow field and heat transfer characteristics in the annulus under different working conditions was studied, and it was concluded by highlighting the following conclusions after comparing the calculation results of different silt wall models.

The flow field in a turbulent Taylor-Couette flow, shear force, turbulent intensity, and radial velocity all showed periodic fluctuations. When fluid in the mainstream region passes through the slit region, under the influence of wall impact and inertial force, a small-scale vortex is formed in the slit region. The vortex energy hence promotes the fluid exchange between the ring gap and the slit region. With an increase in both the number and width of slits, the convection strength between the inner and outer walls is noticed to have increased. Besides, an increase in Reynolds number impacts the slit wall structure significantly influencing the flow field distribution and the heat transfer performance of the model.

Furthermore, an increase in the slit width enhanced the heat transfer capacity of the model due to its effect causing the fluid in the mainstream area to become larger while the position of the impact point becomes shallower. Also, an increase in the number of slits significantly enhanced the heat transfer capacity of the model due to the shallower impact point and the smaller fluid losses due to viscous forces and friction forces. In conclusion, the number and width of slits were both noted to be directly related to the effect on the flow field distribution and heat transfer performance. However, the effect of slit width was more obvious.

\section{ACKNOWLEDGMENT}

This study is supported by the National Natural Science Foundations of China (51676086), Natural Science Foundation of Jiangsu Province (BK20161351), the $15^{\text {th }}$ Six Talents Peak Project of Jiangsu Province (TD-JNHB002), and Jiangsu Provincial Policy Guidance Programme-International Cooperation Programme(BZ2020078).

\section{REFERENCES}

Alziary de Roquefort, T. \& Grillaud, G. 1978. Computation of Taylor vortex flow by a transient implicit method. Computers \& Fluids, 6(4): 256-269.

Coles, D. 1965. Transitions in circular Couette flows. Journal of Fluid Mechanics, 21(3): 385-425.

Fénot, M., Bertin, Y., Dorignac, E., \& Lalizel, G.2011. A review of heat transfer between concentric rotating cylinders with or without axial flow, International Journal of Thermal Sciences, 50(7): 1138-1155.

Gollub, J.P. \& Swinney, H.L. 1975. Onset of turbulence in a rotating fluid. Physical Review Letters, 35: 927-930.

Girimaji, S.S. 2006. Partially-averaged Navier-Stokes model for turbulence: A Reynolds-averaged Navier-Stokes to direct numerical simulation bridging method. Journal of Applied Mechanics, 73(3): 413-421.

Hayase, T., Humphrey, J., \& Greif, R. 1992. Numerical calculation of convective heat transfer between rotating coaxial cylinders with periodically embedded cavities, Journal of Heat Transfer,114: 589-597.

Jeng, T.M., Tzeng, S.C., Wang, Y.C., \& Lin, C.H. 2008. Thermal characteristics in the annulus with an inner rotating rib-roughness cylinder. Heat \& Mass Transfer, 44(5): 527-533.

Kreith F. 1969. Convection heat transfer in rotating systems, Advances in Heat Transfer, 5: 129-251

Lopez, J. M., Marques, F. \& Avila, M. 2015. Conductive and convective heat transfer in fluid flows between differentially heated and rotating cylinders. International Journal of Heat \& Mass Transfer, 90: 959-967. 
Liu, D., Wang, Y.Z., Shi, W. D., Kim, H. B., \& Tang, A. K. 2015. Slit Wall and Heat Transfer Effect on the Taylor Vortex Flow. Energies, 8(3): 1958-1974.

Liu, D., Ding S.C., Zhou, W. J., Sun, S. L., \& Kim, H.B. 2019. Turbulent Taylor-Couette flow and its heat transfer characteristic based on PANS model. Journal of Drainage and Irrigation Machinery Engineering, 37(10): 889-894.

Nouri-Borujerdi, A., \& Nakhchi, M. E. 2017. Optimization of the heat transfer coefficient and pressure drop of Taylor-Couette-Poiseuille flows between an inner rotating cylinder and an outer grooved stationary cylinder. International Journal of Heat and Mass Transfer, 108: 1449-1459.

Ostilla-Mónico, R., Poel, E. P. V. D., \& Verzicco, R. 2014. Exploring the phase diagram of fully turbulent Taylor-Couette flow. Journal of Fluid Mechanics, 761:1-26.

Selimefendigil, F.\& Ztop, H.F. 2014. Forced convection of ferrofluids in a vented cavity with a rotating cylinder, International Journal of Thermal Sciences, 86: 258-275.

Singer, P. H. 1984. Techniques of low pressure chemical vapor deposition. Semiconductor International, 7(5):72-77.

Serre, E., Sprague, M. A., \& Lueptow, R. M. 2008. Stability of Taylor-Couette flow in a finite-length cavity with radial through flow. Physics of Fluids, 20(3): 034106.

Tachibana, F., Fukui, S., \& Mitsumura, H. 1960. Heat transfer in an annulus with inner rotating cylinder. Jsme International Journal, 3(9):119-123.

Vivès, C. 1988. Effects of a forced Couette flow during the controlled solidification of a pure metal. International Journal of Heat and Mass Transfer, 31 (10): 2047-2062.

Yong, N. L., \& Minkowycz, W. J. 1989. Heat transfer characteristics of the annulus of twocoaxial cylinders with one cylinder rotating, International Journal of Heat \& Mass Transfer, 32(4):711-722.

Zhu, X., R Ostilla-Mónico, Verzicco, R., \& D Lohse.2016. Direct numerical simulation of Taylor-Couette flow with grooved walls: torque scaling and flow structure. Journal of Fluid Mechanics, 794: 746-774. 